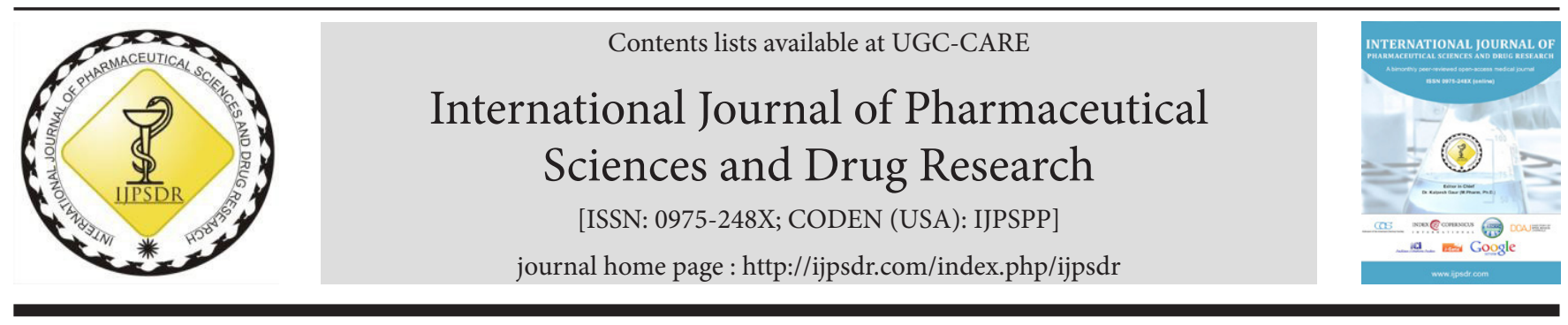

Research Article

\title{
Screening of In vitro Antiviral Activity of Purified Terpenoid Extracts of Selected Seaweeds against Chikungunya Virus
}

\author{
Sabira Siraj Sumayya ${ }^{1}$, Abdulhadeef Shereefa Lubaina ${ }^{2}$, Kumaraswamy Murugan ${ }^{3 *}$ \\ ${ }^{1}$ Sree Neelakanta Government Sanskrit College Pattambi, Palakkad-679306, Kerala, India \\ ${ }^{2}$ Christian College, Kattakada-695572, Kerala, India \\ ${ }^{3}$ Center for Innovation in Science and Social Action (CISSA), Thiruvananthapuram-695010, Kerala, India
}

\begin{tabular}{l} 
A R T I C L E I N F O \\
\hline Article history: \\
Received: 27 March, 2020 \\
Revised: 11 June, 2020 \\
Accepted: 17 June, 2020 \\
Published: 30 July, 2020 \\
Keywords: \\
Antiviral, \\
Chikungunya virus, \\
Purification, \\
Seaweeds, \\
Terpenoid extracts. \\
D0I: \\
10.25004/IJPSDR.2020.120403
\end{tabular}

\section{IN TRODUCTION}

The CHIKV, a re-emerging arbovirus, leads to crippling musculoskeletal inflammatory disorders in humans featured by high fever, polyarthralgia, myalgia, rash, and headache. CHIKV is carried via the Aedes mosquito vector and is capable of an out breaking epidemic, urban transmission cycle with intensified infection rates. Since 2004 , CHIKV has spread to various countries, causing the disease on a global scale, and the potential for its epidemics still remains high. No proven vaccines

\begin{abstract}
A B S T R A C T
Currently, the search for novel phytochemicals with unique biological potentialities is a prerequisite for designing ideal drugs for the humankind. Seaweeds are bioresources with a broad spectrum of medicinal properties with minimal side effects. Kerala, the southern state of India, reported high incidence of chikungunya virus (CHIKV) infections in the last several years. No specific virucidal therapy or effective vaccines are available. This emphasizes the need to search for phytochemicals as drugs with less cost and more effective. Therefore, an attempt was made in screening purified terpenoid extracts of selected seaweeds as anti-CHIKV potential. In this study, the terpenoids composition from the red algae Hypnea musciformis, Kappaphycus alvarezii, and Gracillaria dura were identified and analyzed by thin-layer chromatography and gas chromatography-mass spectrum. The methanolic extract of seaweeds was purified by column chromatography, and each fraction was eluted by using petroleum ether and ethyl acetate as solvent combination. The analysis of the purified fraction of $H$. musciformis and K. alvarezii revealed the presence of several terpenoids and G. dura showed only four major terpenoids, respectively. Vero cell lines were employed in the antiviral assays, infected to CHIKV, and treated with varied doses of purified terpenoid extracts. In the antiviral activity, terpenoid extracts of $G$. dura showed remarkable and promising $\mathrm{EC}_{50}$ inhibitory effect at $1.25 \mu \mathrm{g} / \mathrm{mL}$. Further, the terpenoid extracts displayed efficient virucidal activity against CHIKV (inhibit around 90\%) with $5 \mu \mathrm{g} / \mathrm{mL}$ dosage. As the last phase, terpenoid extracts added at time intervals of $0,1,2$, and 3 post-infection periods still maintained a significant inhibitory potential against CHIKV viral replication. Thus, the overall study suggests that the terpenoid extracts of G. dura may be effectively used in the prevention and treatment of CHIKV infections. Clinical studies may be warranted for designing a promising new anti-CHIKV drug.
\end{abstract}

\footnotetext{
*Corresponding Author: Kumaraswamy Murugan

Address: Center for Innovation in Science and Social Action (CISSA), Thiruvananthapuram-695010, Kerala, India

Email $\square$ : harimurukan@gmail.com

Relevant conflicts of interest/financial disclosures: The authors declare that the research was conducted in the absence of any commercial or financial relationships that could be construed as a potential conflict of interest.

Copyright (c) 2020 Sabira Siraj Sumayya et al. This is an open access article distributed under the terms of the Creative Commons AttributionNonCommercial-ShareAlike 4.0 International License which allows others to remix, tweak, and build upon the work non-commercially, as long as the author is credited and the new creations are licensed under the identical terms.
} 
Sabira Siraj Sumayya et al.

African. ${ }^{[2]}$ West African strains have been linked mainly with enzoonotic transmission and small focal outbreaks of human disease in countries located in western Africa. In contrast, strains from East/ Central/ South African branch have repeatedly spread to new zones to cause remarkable urban epidemics. The first emergence of an East/ Central/ South African strain outside of Africa was noted to have occurred between 70 and 150 years ago in Asia. ${ }^{[3]}$ The virus continued to circulate in this region, evolving independently of the East/ Central/ South African category into a distinct Asian genotype, which results in numerous CHIKV disease outbreaks in this region.

India was impacted by the major outbreak of chikungunya fever during 2006-2007. Kerala, the worst affected state with a contribution of 55.8\%. However, no systematic information is available on infection status of CHIKV in the region. Hence, a post-epidemic survey was carried to estimate seroprevalence status [immunoglobulin G (IgG)] in the community using indirect immune-fluorescence test that is specific and sensitive for CHIKV infection. Mid-highlands region which harbor rubber plantations evidenced $68 \%$ of the population to be seropositive for CHIKV IgG. This may be due to high occupational risk of the male population engaged in plantation activities exposed to mosquito infective bites. The current study provides an insight into screening of efficacy of purified terpenoid extracts from selected seaweeds against CHIKV.

\section{Materials AND METhods}

The marine algae $H$. musciformis, G. dura, and K. alvarezii were collected during March 2018, from the Mandapam coast (latitude $9^{\circ} 17^{\prime} \mathrm{N}$, longitude $79^{\circ} 22^{\prime} \mathrm{E}$ ), Gulf of Mannar. Identity was confirmed by referring algal flora and substantiated by matching with samples of CMFRI, Kochi. The thalli of K. alvarezii, H. musciformis, and $G$. dura were washed thoroughly until all the debris was removed. Then, macerated into pieces, shade dried, and powdered mechanically. Initially, 50 grams of each of the dried algal powder was subjected to Soxhlet extraction with $250 \mathrm{~mL}$ of methanol. The extraction was repeated thrice. The extract was then filtered and kept at room temperature for evaporation. Purification of each sample was done by using silica gel column chromatography (CC). Each fraction was eluted by using different combinations of petroleum ether:ethyl acetate solvents. The column eluted fractions were analyzed for the presence of terpenoids and subjected to thin layer chromatography (TLC), and further determined by gas chromatography-mass spectrometry (GC-MS).

\section{GC-MS Analysis}

For GC-MS analysis, the sample was injected into an HP-5 column $(30 \mathrm{~m} \times 0.25 \mathrm{~mm}$ i.d. with 0.25 ìM film thickness), Agilent Technologies 6890 N JEOL GC Mate II GC-MS model. Helium was used as carrier gas with a flow rate of $1 \mathrm{~mL} / \mathrm{min}$. A chromatogram was obtained, and the mass spectrum of the unknown components was identified by comparing it with the spectrum of the known components available in the NIST library.

\section{Cytotoxicity Assay}

The cytotoxic effect of purified terpenoid extracts from selected seaweeds was analyzed on Vero cells with $90 \%$ confluence in 96 well plates. The cells were pre-treated with $1,2,4,6,8,10$, and $12 \mathrm{mg} / \mathrm{mL}$ of purified terpenoid extracts from selected seaweeds and incubated for 48 to 72 hours in Dulbecco's modified eagle medium (DMEM) and 5\% of fetal bovine serum (FBS) at $37^{\circ} \mathrm{C}$ in $\mathrm{CO}_{2}$ incubator. Further, $5 \mathrm{mg} /$ $\mathrm{mL}$ 3-(4,5-dimethylthiazol-2-yl)-2,5-diphenyltetrazolium bromide (MTT, Sigma-Aldrich) was mixed after 72 hours post-terpenoid extracts treatment and incubated for 3 hours at $37^{\circ} \mathrm{C}$. Gently, the medium was removed, followed by the addition of $100 \mu \mathrm{M}$ dimethyl sulfoxide (DMSO), and cells were incubated at $37^{\circ} \mathrm{C}$ for 15 minutes to dissolve the formazan crystals as prescribed. ${ }^{[4]}$ The optical density (OD) was recorded at $550 \mathrm{~nm}$ using ELISA plate reader. The live active cell \% was compared with the cell's control, and cellular cytotoxicity was calculated. Three independent experimental was carried to substantiate the results.

\section{Antiviral Activity by Virus Plaque Reduction Protocol}

Antiviral potentiality was validated using a virus plaque reduction protocol. Vero cell lines were cultured on 24 well plates under prescribed environment and subsequently infected with CHIKV [multiplicity of infection (MOI) $=0.1$ ] with or without the varied doses of purified terpenoid extracts $(0.625,1.25,2.5,5,10$, and $20 \mu \mathrm{g} / \mathrm{mL})$ at $37^{\circ} \mathrm{C}$. After 1-hour of adsorption, the residual inoculum was changed by medium containing methylcellulose (1\%) and the corresponding dose of each compound. Plaques were counted after 72 hours of incubation at $37^{\circ} \mathrm{C}$ at $5 \% \mathrm{CO}_{2}$. The inhibitory concentration $50 \%\left(\mathrm{EC}_{50}\right)$ was measured as the terpenoid dose required to reduce virus plaques by $50 \%$. All experimental were carried twice and each in triplicate. This was employed to evaluate the number of plaques forming virus units (PFU/mL) compared to mock CHIKV control. ${ }^{[5]}$

\section{Virucidal Profile}

This part of the experiment was carried to assess efficacy of terpenoid extract that has a direct effect on the infectivity of CHIKV. Approximately 200 plaque-forming units of the viruses were incubated at $37^{\circ} \mathrm{C}$ in DMEM containing concentration of $5 \mu \mathrm{g} / \mathrm{mL}$ of terpenoid extract. As the control, the same amount of virus was incubated in DMEM containing 1\% DMSO, and a similar condition was also made for ribavirin. The remaining virus titer obtained after each experiment was determined by plaque assay on Vero cells, as described above. ${ }^{[5]}$

\section{Time Interval of Drug Addition}

The effects of treatment at different time periods were tested to analyze the period of the viral life cycle that was 
affected by the terpenoid extract. Monolayers of Vero cells were cultured on 24 well plates as previously narrated and were infected with CHIKV at an MOI of 0.1 at time period of 0 . Cells were treated with terpenoid extract of G. dura to a dose of $5 \mu \mathrm{g} / 0,1,2,4,6,8,12$, and 16 hours. After 12 hours of incubation, the culture supernatants were collected, and the cells were topped with $3 \%$ carboxy methyl (CM) cellulose in culture medium. After 72 hours of incubation at $37^{\circ} \mathrm{C}$, the cells were fixed with $20 \%$ formaldehyde for 2 hours, and stained with crystal violet for 5 minutes. Plaques formed after each treatment was counted, and titer value was calculated. ${ }^{[5]}$

\section{Statistical Analysis}

The cytotoxicity (CC50) as well as the effective minimal cytotoxic concentration and $\mathrm{IC}_{50}$ of each terpenoid extract, were assessed from dose-response curve (Prism, Graph Pad). Results were compared using Student's t test. The statistical significance level was set at $p$-values $<0.05$.

\section{RESULTS AND DiscuSSION}

\section{GC-MS Analysis}

The crude methanolic extracts of $H$. musciformis, $K$. alvarezii, and $G$. dura were purified by using silica gel G (230-400 mesh size) column chromatography. Each fraction was eluted using petroleum ether:ethyl acetate solvent combinations. Elution was started with $100 \%$ petroleum ether (PE) and gradually increased the polarity up to $100 \%$ Ethyl Acetate (EA). The column eluted fractions were analyzed for the presence of terpenoids and subjected to TLC using $6 \mathrm{~mL}$ toluene: $1 \mathrm{~mL}$ ethyl acetate as solvent combination and further to GC-MS analysis. The fraction eluted using 95:5 solvent combinations of $H$. musciformis, 50:50 solvent combinations of K. alvarezii, and 90:10 of G. dura showed significant amount of terpenoids.

The 95:5 PE:EA eluted fractions of $\mathrm{H}$. musciformis showed a single band on TLC with Rf value 0.89 . The isolated band was subjected to GC-MS revealed eight terpenoid compounds, such as, eicosane, heneicosane, 2-pentadecnone, hexadecanoic acid methyl ester, n-hexadecanoic acid, hexadecanoic acid ethyl ester, heptadecanoic acid methyl ester, 11-octadecanoic acid methyl ester. 50:50 solvent combinations of $K$. alvarezii showed two bands of terpenoids and its GC-MS analysis showed the presence of hexadecane, eicosane, heptadecane, octadecane, heneicosane, tricosane, hexadecanoic acid, methyl ester, and beta amyrin. The 90:10 (PE:EA) purified fraction of $G$. dura also showed single prominent band on TLC (Rf values 0.47 ) and revealed the presence of terpenoid fractions, such as, hexadecanoic acid methyl ester, n-hexadecanoic acid, 11-octadecanoic acid, and phytol.

\section{Evaluation of the Cytotoxicity}

The $\mathrm{CC}_{50}$ of the purified terpenoid extracts from the red algae G. dura, K. alvarezii, and H. musciformis were evaluated by MTT reagent. The results in Table 1 reveal the significant $\mathrm{CC}_{50}$ value showed by $G$. dura as compared to the other seaweeds, such as, K. alvarezii and H. musciformis, i.e., $6 \mathrm{mg} / \mathrm{mL}$. Subsequently, terpenoid extract of the seaweeds were evaluated further for their virucidal potentialities.

$\mathrm{CC}_{50}$ is the $50 \%$ cy totoxic concentration, the dose that reduces cell viability by $50 \%$ compared to control.

$\mathrm{EC}_{50}$ is the dose that reduced CHIKV replication by $50 \%$, compared to infected control.

Selectivity index the ratio between $\mathrm{CC}_{50}$ and $\mathrm{EC}_{50}$, and represents the safety for in vitro assays.

\section{Anti-CHIKV Profile and Selectivity Index}

For this analysis, different dosages of the terpenoid extracts $(0.625$ to $20 \mu \mathrm{g} / \mathrm{mL})$ from G. dura, K. alvarezii, and $H$. musciformis were used. Purified terpenoid extracts from $G$. dura showed a concentration-dependent \% inhibition, i.e., $90 \%$ inhibition at $5 \mu \mathrm{g} / \mathrm{mL}$ concentration, $96 \%$ inhibition at $10 \mu \mathrm{g} / \mathrm{mL}$, and $98.45 \%$ inhibition at $20 \mu \mathrm{g} / \mathrm{mL}$ extract. The $\mathrm{EC}_{50}$ value of the purified terpenoid extract from $G$. dura was $1.25 \mu \mathrm{g} / \mathrm{mL}$. The obtained data suggest that terpenoid extracts from $G$. dura were able to inhibit the replication of CHIKV by inhibiting plaque formation at various concentrations steadily (Fig. 1). Based on the \% inhibition results, the selectivity index (SI) was evaluated that reflects the degree of reliability of the drug for future plausible usage and that reflects the relationship between $\mathrm{CC}_{50}$ and $\mathrm{EC}_{50}$, corroborating the perspectives that the extract with the lowest $\mathrm{EC}_{50}$ also had a higher index of selectivity. Purified terpenoid extracts of $G$. dura presented a remarkable index of 4,800 (Table 1).

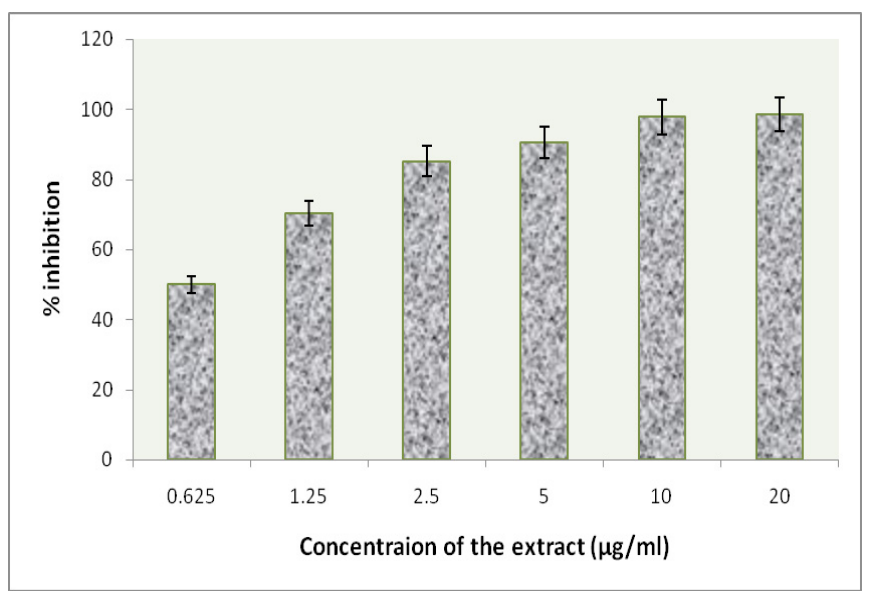

Fig. 1: Effect of the terpenoids of G. dura on CHIKV replication at different concentrations $0.625,1.25,2.5,5,10$, and $20 \mu \mathrm{g} / \mathrm{mL}$

Table 1: Cytotoxicity, anti-CHIKV profile, and selectivity index of terpenoid extracts from the seaweeds

\begin{tabular}{llll}
\hline Terpenoids & $C C_{50}(\mathrm{mg} / \mathrm{mL})$ & $E C_{50}(\mu \mathrm{g} / \mathrm{mL})$ & $S I$ \\
\hline G. dura & 6 & 1.25 & 4,800 \\
H. musciformis & 8 & - & - \\
K. alvarezii & 10 & - & - \\
Ribavirin 297 & 0.25 & 2.5 & 100 \\
\hline
\end{tabular}


Esteves et al. ${ }^{[5]}$ demonstrated that the $\mathrm{CC}_{50}$ value of green algal extract of Caulerpa racemosa was $589.3 \mu \mathrm{g} / \mathrm{mL}$. CHIKV is an arbovirus transferred to humans by Aedes mosquitoes. The virus penetrates the skin and enters the bloodstream. After the initial replication within dermal fibroblasts, it spreads via the bloodstream to the liver, muscles, joints, spleen, lymph nodes, and brain. The infection is featured by severe joint pain of abrupt onset, intense fever, and rash. Non-specific clinical symptoms are cervical adenopathy, asthenia, chills, nausea, vomiting, and diarrhea. ${ }^{[6]}$ Many studies have been carried out in search of novel drugs that can react to viral replication, decreasing the severity of the cases, and rejuvenating the health of the infected people.

For the last several years, strategies using secondary metabolites of marine weeds or from other biosources and their derivatives become popular mainly due to their poor toxicity and their potent activity against different viral strains as previously described. ${ }^{[7-9]}$ The present study revealed that the terpenoids of $G$. dura has less toxicity with a $\mathrm{CC}_{50}$ value of $6 \mathrm{mg} / \mathrm{mL}$, demonstrating that this molecule can be an ideal promising drug for trail. Similarly, they exhibit remarkable virucidal activity, i.e., the $\mathrm{EC}_{50}$ value of CHIKV was $1.25 \mu \mathrm{g} / \mathrm{mL}$ and reflecting a SI of 4,800 .

\section{Evaluation of the Virucidal Potential}

This part of the experiment was carried by evaluating the plaque formation and also the viral titer quantification. As shown in Fig. 1, terpenoids of $G$. dura were capable of arresting the CHIKV replication in $97.89 \%$ at the dosage of $5 \mu \mathrm{g} / \mathrm{mL}$. However, the positive reference drug ribavirin yielded a lower value only, i.e., 95\% of CHIKV virucidal activity.

Generally, the antiviral potential of drugs is evaluated by determining their potency in regulating epidemic outbreaks, i.e., by monitoring the drug as a preventive measure. The present data showed a concentration-dependent antiviral activity of inhibition

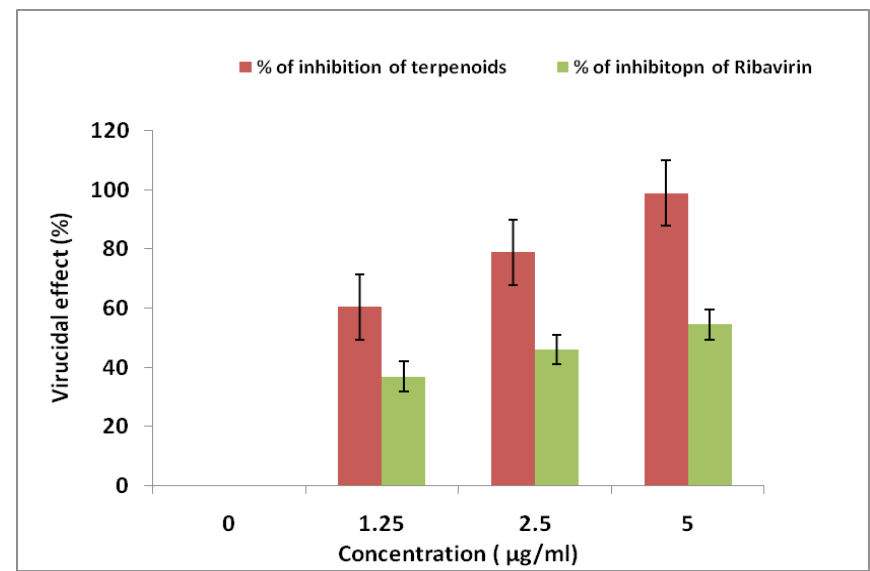

Fig. 2: Effect of terpenoid extract from $G$. dura on the infectivity of CHIKV; Vero cells were infected with CHIKV at an MOI of 0.1; the results were evaluated by plaque assay; error bars indicate that the standard deviation up to $100 \%$ of CHIKV activity at the dosage of $5 \mu \mathrm{g} / \mathrm{mL}$ used (Fig. 2).

\section{Time of Addition Assay}

Further, the impact of the terpenoids at different time intervals of addition on Vero cell lines infected by CHIKV was evaluated. The terpenoids were added at $\mathrm{T}=0,1,2$, and 3 hours (simultaneous) had a statistically remarkable inhibitory effect, i.e., sound inhibition rate on the viral particle and was comparable with the drug ribavirin (a sharp decline of its activity after 8 hours, meanwhile terpenoids displayed a decline accentuated even after 12 hours, as shown in the Fig. 3.

The determination of the mode of action is a strategy to substantiate whether the compound has a promising role and also to reveal its unique specificities. The search for phytochemicals that act at various stages of replication of the virus, in addition to its poor toxicity may be ideal for determining its possibility to adopt for clinical trial. The observed the poor cytotoxicity and sound inhibition of the CHIKV replication and the inhibitory role of the terpenoids added at various time intervals after viral infection suggests the above said direction. The noted data on addition of the molecule in the time intervals $0,1,2$, and 3 hours post-infection stage still maintained an inhibitory potential of viral replication to nearly $100 \%$ for CHIKV. Parsania et al. ${ }^{[10]}$ screened antiviral activities of four plant extracts against acyclovir-resistant herpes simplex virus type-1. The antiviral effects of Mentha pulegium extract on HSV-1 were more remarkable than the virucidal potentiality. Visintini et al. ${ }^{[11]}$ established the effective Bidens gaudichaudiana as the most promising species in terms of in vitro antiviral activity among the herbal extracts of Asteraceae species. Kohn et al. ${ }^{[12]}$ analyzed in vitro antiviral activities of plants from Brazilian cerrado against the avian metapneumovirus (aMPV). Montanha et al. ${ }^{[13]}$ screened virucidal activities of Brazilian herbal extracts. Lelesius et al. ${ }^{[14]}$ documented in vitro antiviral activity of fifteen plant extracts against avian infectious bronchitis virus. Ahmad et al. ${ }^{[15]}$ explored

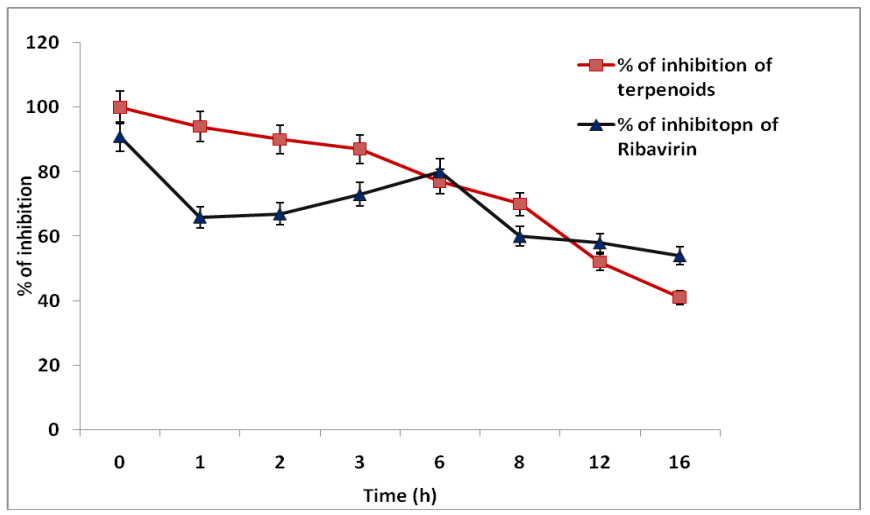

Fig. 3: Time course chart showing different treatment times (hr) at the concentration of $5 \mu \mathrm{g} / \mathrm{mL}$ with the action of the drugs in infection 
in vitro cytotoxic and antiviral activities of different medicinal plants against infectious bursal disease (IBD) virus of Central Europe. Tello et al. ${ }^{[16]}$ proved cytotoxic, virucidal, and antiviral activity of South American plant species and algal extracts. Rosmalena et al. ${ }^{[17]}$ experimented with the antiviral effect of Indonesian medicinal plant extracts by in vitro and in silico condition against dengue virus. Hudson and Vimalanathan ${ }^{[18]}$ analyzed the phytochemicals of Echinacea purpurea under light-dependent and light-independent antiviral activities. Todorov et al. ${ }^{[19]}$ confirmed the antiviral activity of Nepeta nuda. Generally, the CHIKV replication cycle is approximately 6 hours. ${ }^{[20]}$ However, many reported reviews demonstrating the drug candidates that function at various periods of the replication cycle, but with an uncertainty of therapeutic efficacy. Further, the present study also demonstrated the efficacy of terpenoid via its antiviral activity, including the time scale potentiality.

The present results showed that the terpenoid extracted from $G$. dura displayed activity against CHIKV. With regard to the possible mode of action of terpenoid revealed sound virucidal pattern. The data tempted for searching the marine resources for antiviral drugs with activity against various viral strains that can remarkably decline the morbidity and mortality of infection. Virucidal activity of purified terpenoid extracts may be useful in developing a preventive strategy to effectively reduce the emergence of new pandemic issues.

\section{REFERENCES}

1. Broeckel R, Haese N, Messaoudi I, Streblow DN. Nonhuman primate models of chikungunya virus infection and disease (CHIKV NHP Model). Pathogens. 2015;4:662-681.

2. Volk SM, Chen R, Tsetsarkin KA, Adams AP, Garcia TI, Sall AA, et al. Genome-scale phylogenetic analyses of chikungunya virus reveal independent emergences of recent epidemics and various evolutionary rates. J Virol. 2010;13:6497-6504.

3. Schuffenecker I, Iteman I, Michault A, Murri S, Frangeul L, Vaney M, et al. Genome microevolution of chikungunya viruses causing the Indian Ocean outbreak. PLoS Med. 2006;7:263-271.

4. Shafiee F, Sadeghi-Aliabadi H, Hassanzadeh F. Evaluation of cytotoxic effects of several novel tetralin derivatives against HeLa, MDA-MB-468, and MCF-7 cancer cells. Adv. Biomed. Res.
2012;1:76-81.

5. Esteves PO, de Oliveira MC, de Souza Barros C, Cirne-Santos CC, Laneuvlille VT, Palmer Paixao IC. Antiviral effect of caulerpin against chikungunya. Nat Prod Commun. 2019;14:1-6.

6. Cunha RVD, Trinta KS. Chikungunya virus: clinical aspects and treatment-a review. Mem Inst Oswaldo Cruz. 2017;112:523-531.

7. Sengupta S, Mukherjee S, Haldar S, Bhattacharya N, Tripathi A. Re-emergence of Chikungunya virus infection in Eastern India. Braz J Microbiol. 2020;51:177-182.

8. Aziz Bamogo PK, Brugidou C, Sereme D, Tiendrebeogo F, Djigma FW, Simpore J, et al. Virus-based pharmaceutical production in plants: an opportunity to reduce health problems in Africa. Vir J. 2019;16:167-172.

9. Esposito DL, Fonseca BA. Zika and chikungunya infections in Brazil: reviewing the epidemic and treatment options. Bras Med Trop. 2016;49:535-536.

10. Parsania M, Rezaee M, Monavari SH, Jaimand K, Mousavi Jazayeri SM, Razazian M, et al. Antiviral screening of four plant extracts against acyclovir resistant herpes simplex virus type-1. Pak. J. Pharm. Sci. 2017;4:1407-1411.

11. Visintini Jaime MF, Redko F, Muschietti LV, Campos RH, Martino VS, Cavallaro LV. In vitro antiviral activity of plant extracts from Asteraceae medicinal plants. Virology J. 2013;10:1-10.

12. Kohn LK, Foglio MA, Rodrigues RA, Sousa IM de O, Martini MC, Padilla MA, et al. In vitro Antiviral Activities of Extracts of Plants of The Brazilian Cerrado against the Avian Metapneumovirus (aMPV). Braz J of Poul Sci. 2015;3:275-280.

13. Montanha JA, Moellerke P, Bordignon SAL, Schenkel EP, Roehe PM. Antiviral Activity of Brazilian Plant Extracts. Acta Farm. Bonaerense. 2004;2:183-186.

14. Lelesius R, Karpovaite A, Mickiene R, Drevinskas T, Tiso N, Ragazinskiene 0 , et al. In vitro antiviral activity of fifteen plant extracts against avian infectious bronchitis virus. BMC Vet Res. 2019;15:1-10.

15. Ahmad W, Ejaz S, Anwar K, Ashraf M. Exploration of the in vitro cytotoxic and antiviral activities of different medicinal plants against infectious bursal disease (IBD) virus Cent. Eur J Biol. 2014;5:531-542.

16. Tello P, Mirazo S, Dutra C, P'erez A, Geis-Asteggiante L, Frabasile S, et al. The antiviral effect of Indonesian medicinal plant extracts against dengue virus in vitro and in silico. Pathogens. 2019;8: 85-92.

17. Hudson JB and Vimalanathan S. The phytomedicine Echinacea purpurea contains light dependent and light-independent antiviral activities. J of Innovat in Pharma and Biol Sci. 2017;4:109-113.

18. Todorov D, Shishkova K, Dragolova D, Hinkov A, Kapchina-Toteva V, Shishkov S. Antiviral activity of medicinal plant Nepeta nuda. Biotech and Biotechnological Equi. 2015;29:39-43.

19. Silva LA, Dermody TS. Chikungunya virus: epidemiology, replication, disease mechanisms, and prospective intervention strategies. J Clin Invest. 2017;127:737-749. 